\title{
Leaf Identification Method Based on BP Neural Network Peng Huang ${ }^{1, a}$ and Zhiliang Kang ${ }^{1, b^{*}}$
}

${ }^{1}$ College of Mechanical and Electronic Engineering, Sichuan Agricultural University, Ya'an, 625014, China

ahpsjdyd@qq.com, 'Zhiliangkang96@163.com

* The Corresponding Author

Keywords: BP neural network; Gray-level co-occurrence matrix(GLCM); Texture features; Leaf shape features

\begin{abstract}
In view of the time-consuming and laborious disadvantages of traditional tree classification methods, the leaf identification method based on BP neural network is established in this paper. The leaves of osmanthus tree, ficus virens and ficus concinna were taken as objects. First, three leaf samples were collected, and then after image preprocessing, 19 feature parameters were extracted, including 3 leaf shape features and 16 texture features based on gray-level co-occurrence matrix(GLCM). Finally, the BP neural network was used to classify and identify the above three kinds of leaves. The simulation results showed that this method can classify and identify the leaves of osmanthus tree, ficus virens and ficus concinna quickly and accurately.
\end{abstract}

\section{Introduction}

The identification and classification of leaves are to distinguish the species of leaves and explore the origin of leaves, which is of great importance to the development of human beings and popularization of science [1-3]. At present, the identification and classification of leaves are mainly done by people. However, it is impossible for any botanist to know the species of all leaves in the face of a large leaf world, which makes it difficult to further study the leaves. With the continuous development of computer image processing technology, it provides a great convenience for the description and quantitative analysis of leaf structure.

GLCM is a simple, fast and effective method for extracting image features [4, 5]. The attribute value of the GLCM of the image obtained by statistics is used as its feature vector. The feature vector can be regarded as a description of the texture feature of the image, which can be used for classification, recognition and retrieval. In this paper, we first pretreat the collected images, extract the leaf texture features based on GLCM, and then classify and identify three kinds of leaves of osmanthus tree, ficus virens and ficus concinna by using BP neural network.

\section{Image Preprocessing}

In this paper, three kinds of leaves of osmanthus tree, ficus virens and ficus concinna were selected. These three kinds of leaves are very similar in form, so it is difficult to distinguish them. All leaf images were photographed under indoor lighting, with 200 samples of each leaf, with a total of 600 samples.
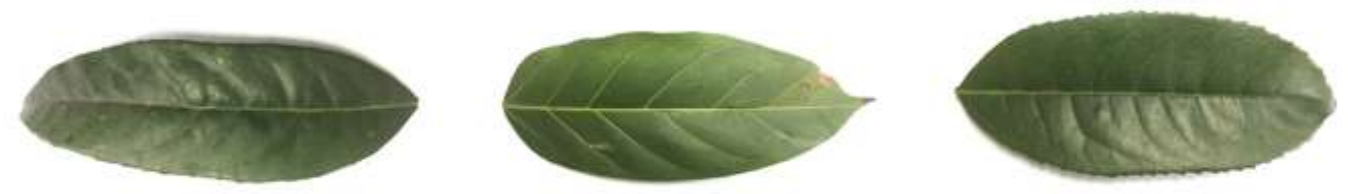

Figure 1. Finite Osmanthus tree, ficus virens and ficus concinna samples 
Graying Processing of Leaf Blade Image. This not only does not reduce the corresponding characteristic information of the leaf blade, but also reduces the calculation amount, and optimizes the efficiency and accuracy of the identification of the leaf blade type[6]. Our eyes are insensitive to blue and are rather sensitive to green. According to the importance and other reference target values, the weighted average of R, G, and B is calculated according to the different weights. The formula is as follows:

$$
\operatorname{Gray}(i, j)=0.299 * R(i, j)+0.578 * G(i, j)+0.114 * B(i, j)
$$

Denoising of Leaf Blade Image. Leaf images are often affected by a variety of noises from the outside when extracting. These noises will affect the quality of the image accordingly, and they will also be displayed on the image, with a certain gray value. The pixel points of the noise are obviously different from those of some other pixels. The median smoothing filter is to take the median value of several pixels in the vicinity, and take it as the gray value of this point. In that way, the image will be more smooth. The median smoothing filter can not only achieve the effect of eliminating noise interference, but also can not change the original information of the image so as to achieve satisfactory recovery results.

Binarization of Leaf Blade Image. Binarization processing is a necessary step for feature extraction and image segmentation [7]. In binarization processing of leaf blade image, the most indispensable step is to define the grayscale threshold of the leaf blade image. If the gray value of the unit on the image is greater than the gray threshold of the defined leaf blade image, the unit value is set to 1 . If the gray value of the unit on the image is smaller than the gray threshold of the defined leaf blade image, the unit value is set to 0 . As a result, the grayscale image is changed into a required binarization image.

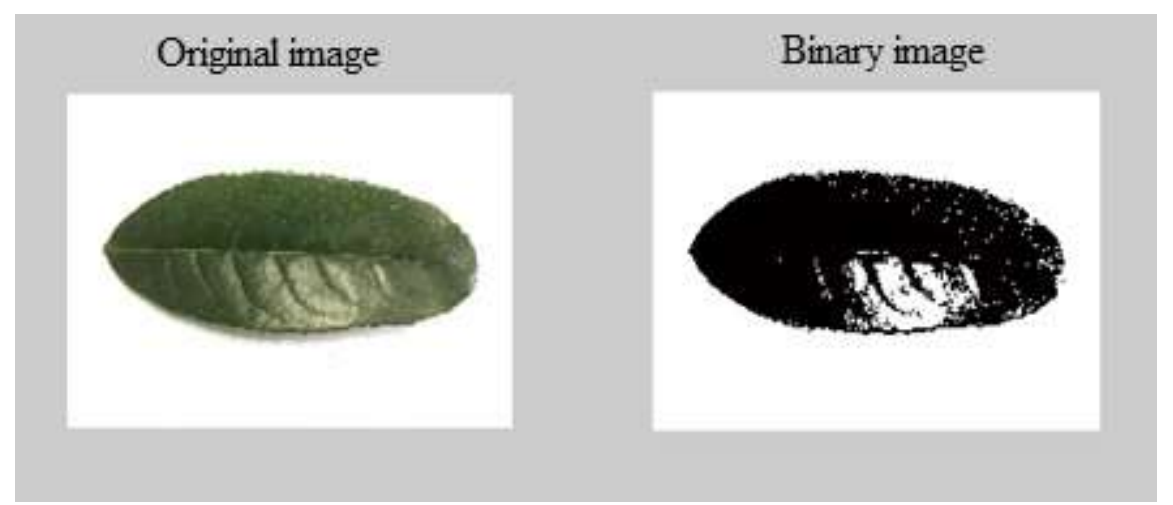

Figure 2. Finite Sample binaryzation of ficus concinna No.1

\section{Feature Extraction}

After a series of related preprocessing, 19 feature parameters were extracted, including 3 leaf shape features and 16 texture features based on GLCM.

The Texture Feature Extraction Based on GLCM. The GLCM is first to obtain the grayscale image of the image through preprocessing, and then calculate the co-occurrence matrix of the image through the grayscale image. Through this co-occurrence matrix, some characteristic parameters of the matrix can be obtained, which can be used to represent the texture features of the image. The GLCM can reflect the comprehensive information about the direction, adjacent interval and variation amplitude of the image grayscale, and it is the basis for analyzing the local patterns of images and their arrangement rules. The main texture features extracted in this paper are as follows:

The first is the second moment(energy).

$$
A S M=\sum_{i=1}^{k} \sum_{j=1}^{k}(G(i, j))^{2}
$$

The second moment is the sum of the squares of the element values of all pixel poin ts in GLCM, also known as energy. 
The second is the contrast.

$$
C O N=\sum_{n=0}^{k-1} n^{2}\left\{\sum_{|i-j|=n} G(i, j)\right\}
$$

The contrast is to calculate the distribution of the element values of all pixel points $i$

$n$ GLCM and the local changes in the image.

The third is the relevance (homogeneity).

$$
C O R=\sum_{i=1}^{k} \sum_{j=1}^{k} \frac{(i j) G(i, j)-u_{i} u_{j}}{s_{i} s_{j}}
$$

Among them:

$$
\begin{aligned}
& u_{i}=\sum_{i=1}^{k} \sum_{j=1}^{k} i \cdot G(i, j) \\
& u_{j}=\sum_{i=1}^{k} \sum_{j=1}^{k} j \cdot G(i, j) \\
& s_{i}^{2}=\sum_{i=1}^{k} \sum_{j=1}^{k} G(i, j)\left(i-u_{i}\right)^{2} \\
& s_{j}^{2}=\sum_{i=1}^{k} \sum_{j=1}^{k} G(i, j)\left(j-u_{j}\right)^{2}
\end{aligned}
$$

The relevance is used to measure the similarity of elements of GLCM in the direction of row or column, also known as homogeneity.

The fourth is the entropy.

$$
E N T=-\sum_{i=1}^{k} \sum_{j=1}^{k} G(i, j) \log G(i, j)
$$

Entropy is the measure of the amount of information that the image has, and the text ure information is also the information of the image. It is a random measure.

\section{Extraction of Leaf Shape Features of the Leaf Blade.}

The first is the length-width ratio.

The length-width ratio is defined as the ratio of length to width of the minimum bounding rectangle of the leaf blade. The calculation formula of length-width is:

$$
S=\frac{L 1}{L 2}
$$

In the formula, L1 is the length of the minimum bounding rectangle, and the L2 is $\mathrm{t}$ he width of the minimum bounding rectangle.The second is the rectangularity.

The rectangularity refers to the ratio of the leaf blade area to the minimum bounding rectangle area. The calculation formula of the rectangularity is:

$$
R=\frac{S 1}{S 2}
$$

In the formula, $\mathrm{S} 1$ is the area of the leaf blade, $\mathrm{S} 2$ is the area of the minimum boun ding rectangle of the leaf blade.

The third is the density.

Density refers to the compactness of the leaf image, defined in terms of perimeter an d area.

$$
C=\frac{4 \pi S}{Q^{2}}
$$

In the formula, $\mathrm{S}$ is the area of the leaf blade image, $\mathrm{Q}$ is the perimeter of the leaf blade image. 


\section{Leaf Identification Based on BP Neural Network}

The Selection of the Hidden Layer. The selection of the hidden layer of BP neural network is very limited[8,9]. If the number of hidden layers is too large, then the speed of the network training will drop sharply, making the error curve of the BP neural network unstable. In general, the completion of the corresponding mapping requires only one hidden layer. In this paper, the three-layer neural network is used.

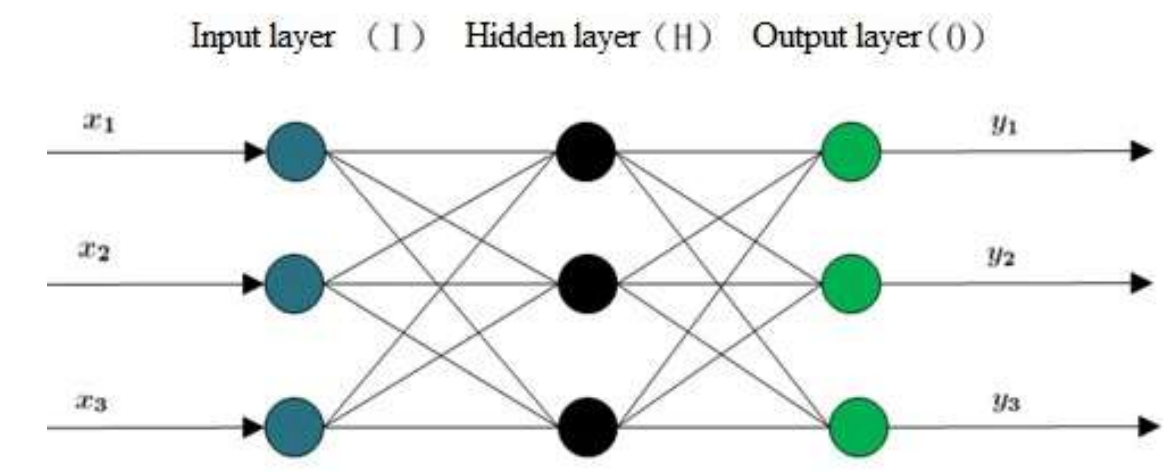

Figure 3. Finite Three layer BP neural network structure diagram

The Selection of the Number of Nodes. For all the collected samples after preprocessing, 19 features, including 16 texture features and 3 leaf blade shape features were extracted. Therefore, the number of the input layer units of the BP neural network was 19. In this paper, the research objects were leaves of osmanthus tree, ficus virens and ficus concinna, so the number of the output layer units was 3. The results of the 3 kinds of output were respectively 100 leaves of osmanthus tree, 010 - leaves of ficus virens and 001 - leaves of ficus concinna. Since there is no definite formula for the number of hidden layer units, the number of hidden layer units can only be adjusted by constant test, and the experimental results are are observed to make a contrast. In this paper, the optimum number of hidden layer units is 5 .

\section{The Establishment of BP Neural Network.}

The first step is to initialize the BP neural network, initialize the weights and deviations of each layer of neurons and set other parameters.

The second step is to determine the input and output of the BP neural network. The extracted leaf image features are used as the input of BP neural network, and the actual output results of each sample are determined at the same time.

The third step is to calculate the output results of the hidden layer and the output layer of the BP neural network.

The fourth step is to compare the theoretical output of the BP neural network with the actual output, and calculate the deviation between the two.

The fifth step is to adjust the weight parameters between each layer of neurons.

Finally, if the training requirement is not met, then go to step 3 and relearn, until the deviation of the target value and the actual output value meets the training accuracy requirement.

\section{Simulation Results}

After the BP neural network was successfully established, three kinds of leaves were classified and identified by BP neural network, and the recognition results reached the expected goal. The training set used 100 samples each, with a total of 300 samples. The test set used 50 samples each, with a total of 150 samples. The leaf recognition results are shown in Table 1. 
Table 1 Identification results of three leaves by BP neural network

\begin{tabular}{|c|c|c|c|c|}
\hline Sample set & Leaf category & Sample number & Recognition results & Recognition rate \\
\hline \multirow{3}{*}{ Test set } & Osmanthus tree & 50 & 49 & $98 \%$ \\
\hline & Ficus virens & 50 & 50 & $100 \%$ \\
\hline & Ficus concinna & 50 & 50 & $100 \%$ \\
\hline
\end{tabular}

\section{Conclusions}

In this paper, in view of the time-consuming and laborious disadvantages of traditional tree classification methods, the leaves of osmanthus tree, ficus virens and ficus concinna were taken as objects to extract the texture features of them based on GLCM and the leaf shape features, and the three kinds of leaves were classified and identified with BP neural network. The experimental results show that the GLCM and the BP neural network have high recognition accuracy and have a certain practical value.

\section{Acknowledgements}

This work was supported by Sichuan agricultural university dual - branch program.

\section{References:}

[1] S. W. Lee and J. S. Kim: Individual Leaf Identification from a Two-Dimensional Monocotyledon Image Based on Phytomorphological Graph Reconstruction, IEEE/RSJ International Conference on Intelligent Robots and Systems, (Vancouver, Canada, September 24-28, 2017), p. 5991.

[2] A. Salman, A. Semwal, U. Bhatt, and V. M. Thakkar: Leaf Classification and Identification Using Canny Edge Detector and SVM Classifier, International Conference on Inventive Systems and Control, (Coimbatore, India, January 19-20, 2017), p. 1.

[3] A. Yadav, M. K. Dutta, C. M. Travieso, and J. B. Alonso: Automatic Identification of Botanical Samples of Leaves Using Computer Vision, International Conference and Workshop on Bioinspired Intelligence (Funchal, Portugal, January 10-12, 2017), p. 1.

[4] L. Dandan, M. Ruixiang, W. Yan, Z. Xianli, and J. Qiao: Multi-scale GLCM Based Quantitative Assessment Method for Ultrasound Liver Fibrosis Images, IEEE 13th International Conference on Signal Processing (Chengdu, China, November 6-10,2016), p. 677.

[5] M. Imani and H. Ghassemian: GLCM, Gabor, and Morphology Profiles Fusion for Hyperspectral Image Classification, Iranian Conference on Electrical Engineering (Shiraz, Iran, May 10-12, 2016), p. 460.

[6] C. Gang: Application of Processing Techniques from Color Image to Grey Image, 2nd International Conference on Software Technology and Engineering, (San Juan, PR, USA, October 3-5, 2010), Vol.2, p. 372.

[7] K. Subramani and S. Murugavalli: A Novel Binarization Method for Degraded Tamil Palm Leaf Images, Eighth International Conference on Advanced Computing (Chennai, India, January 19-21, 2017), p. 176.

[8] Peng L, Shizhao N, Zheng W, Ziwei J, Jianwu Y, Zhongxiang Q, Wangmo P. Predicting durations of online collective actions based on Peaks' heights [J]. Communications in Nonlinear Science and Numerical Simulation. 2018, 55: 338-354.

[9] L. Shao, Y. Mu, J. Liu, G. Dong, H. Liu, and P. Guo: The Trunk of the Image Recognition Based on BP Neural Network, IEEE International Conference on Mechatronics and Automation, (Tianjin, China, August 3-6, 2014), p. 1800.

[10] M. You and Y. Li: Automatic classification of the diabetes retina image based on improved BP neural network, 33rd Chinese Control Conference (Nanjing, China, July, 28-30, 2014), p. 5021. 